\title{
ROOTS OF SCALAR OPERATORS
}

\section{J. G. STAMPFLI}

Introduction. The existence of normal roots of normal operators is a well known consequence of the spectral theorem. Very simple examples show however that normal operators may have roots which are not normal (in fact the identity operator has non-normal roots). In this paper we will show that the invertible scalar operators on a Banach space possess only scalar operators as roots.

Preliminaries. The term operator will be used to mean a bounded linear transformation of a Banach space into itself. By a spectral measure on a Banach space $x$, we mean a family of bounded operators $E(\cdot)$ defined on all Borel sets $\sigma$ of the plane, with the following properties.

(i) $E$ (empty set) $=0, E$ (plane) $=I$, where $I$ is the identity.

(ii) For all $\sigma_{1}, \sigma_{2} ; E\left(\sigma_{1} \cap \sigma_{2}\right)=E\left(\sigma_{1}\right) \cdot E\left(\sigma_{2}\right)$ and for disjoint $\sigma_{1}, \sigma_{2}$

$$
E\left(\sigma_{1} \cup \sigma_{2}\right)=E\left(\sigma_{1}\right)+E\left(\sigma_{2}\right) \text {. }
$$

(iii) There exists a constant $M$ such that

$$
\|E(\sigma)\| \leqq M \quad \text { for all } \sigma .
$$

(iv) For $x \in X$ and $\left\{\sigma_{n}\right\}$ a sequence of disjoint Borel sets,

$$
E\left(\bigcup_{n=1}^{\infty} \sigma_{n}\right) x=\sum_{n=1}^{\infty} E\left(\sigma_{n}\right) x .
$$

If an operator $S$ admits a representation $S=\int z d E(z)$ where $E(\cdot)$ is a spectral measure then $S$ is a scalar operator. $T$ is a spectral operator if $T=S+N$ where $S$ is a scalar operator, $N$ is a quasi-nilpotent operator and $S$ commutes with $N$.

I. Lemma 1. If $T^{n}=I, n$ a positive integer, $I$ the identity on the $B a$ nach space $x$, then $T$ is a scalar operator, of the form $T=\sum_{i=1}^{n} w_{i} E_{i}$ where $E_{i} E_{j}=\delta_{i j} E_{i}, i, j=1, \cdots, n$ and $\sum_{i=1}^{n} E_{i}=I$.

Proof. By Dunford's spectral mapping theorem the spectrum of $T$ can consist of at most the $n$th roots of unity. Then the resolvent $(T-z I)^{-1}$ is holomorphic in the rest of the plane, so by $[3$, p. 179], we have

$$
T=\sum_{i=1}^{n} N_{i}+\sum_{i=1}^{n} w_{i} E_{i}
$$

where the $N_{i}$ s are quasi-nilpotent operators, the $E_{i}$ s are idempotent

Received by the editors March 11, 1961 and, in revised form, August 23, 1961. 
operators and the $w_{i}$ s are the $n$th roots of unity. Also

$$
\begin{gathered}
E_{i} E_{j}=0, \quad i \neq j ; \quad E_{i} N_{i}=N_{i} E_{i}=N_{i} ; \quad E_{i} N_{j}=N_{j} E_{i}=0, \quad i \neq j ; \\
E_{i} T=T E_{i} \text { and } \sum_{i=1}^{n} E_{i}=I .
\end{gathered}
$$

Now we will show all $N_{i}$ s are zero. Multiplying (1) by $E_{j}$ we obtain $T E_{j}=\left(N_{j}+w_{j} I\right) E_{j}$. Raising this to the $n$th power we have $E_{j}$ $=\sum_{i=0}^{n}\left(\begin{array}{l}n \\ i\end{array}\right) w^{n-i} N_{j}^{i} E_{j}$ or

$$
0=\sum_{i=1}^{n}\left(\begin{array}{l}
n \\
i
\end{array}\right) w^{n-i} N_{j}^{i} E_{j}
$$

Now for any element of the form $y=E_{j} x$ we have $\sum_{i=1}^{n}\left(\begin{array}{l}n \\ i\end{array}\right) w^{n-i} N_{j}^{i} y$ $=0$. This implies that $N_{j}^{n} y$ is linearly dependent on $\left\{y, N_{j}^{\prime} y, \cdots, N_{j}^{n-1} y\right\}$ and hence the linear subspace $M$, generated by this set is invariant under $N_{j}$. Since $N_{j}$ is quasi-nilpotent on $\mathbb{X}$, $\left.N_{j}\right|_{M}$ is nilpotent. Assume that $\left.N_{j}\right|_{M} \neq 0$. Then there exists $u \in M$, $u=E_{j} v$ such that $N_{j} u \neq 0, N_{j}^{2} u=0$ because $\left.N_{j}\right|_{M}$ is nilpotent. But then from (2) we have $N_{j} u=0$ contrary to hypothesis. Hence $N_{j} \equiv 0$ on $M$ and since $y$ was chosen arbitrarily from $E_{j} x$, we have $N_{j} E_{j}=0$. Thus $N_{j}=N_{j} \sum_{i=1}^{n} E_{i}=\sum_{i=1}^{n} N_{j} E_{i}=0$. We may now write $T=\sum_{i=1}^{n} w_{i} E_{i}$ and from this conclude that $T$ is a scalar operator. We will now quote Theorem 2 of $[2$, p. 452]:

LEMMA 2. Let $A$ and $B$ be commuting scalar operators on a Banach space $x$ where

$$
B=\sum_{i=1}^{k} z_{i} E_{i} \quad \text { and } \quad E_{i} E_{j}=0, \quad i \neq j ; \quad E_{i} \cdot E_{i}=E_{i} ; \quad \sum_{i=1}^{k} E_{i}=I ;
$$

then $A \cdot B$ is a scalar operator.

II. TheOREM 1. Let $T^{n}=S$, where $n$ is a positive integer and $S$ an invertible scalar operator on the Banach space $x$; then $T$ is a scalar operator.

Proof. Since $S$ is scalar, $S=\int z d F(z)$. Let $A=\int z^{1 / n} d F(z)$ where $z^{1 / n}$ is the principal $n$th root of $z$. Now $A$ is a scalar operator and $A^{n}=S$. Because $T$ commutes with $S, T$ commutes with the spectral measure $F(z)$ and hence $T$ commutes with $A$ (see $\left[1\right.$, p. 329]). Let $T A^{-1}=B$, then $B^{n}=T^{n} A^{-n}=S S^{-1}=I$. By Lemma $1, B$ is a scalar operator of the form $B=\sum_{i=1}^{n} w_{i} E_{i}$; and since $T A=A T$ we have $T=A B=B A$. We may therefore invoke Lemma 2 to conclude that $T$ is a scalar operator.

Corollary 1. Let $T^{n}=S$ where $n$ is a positive integer and $S$ is a 
scalar operator on the Banach space $X$ with zero an isolated point of the spectrum of $S$; then $T=S_{1}+N$ where $S_{1}$ is scalar, $N$ is nilpotent, $S_{1}$ commutes with $N$ and $N^{n}=0$. Thus $T$ is a spectral operator.

The proof is obtained by a slight modification in the proof of Theorem 1.

The author would like to express his gratitude to Charles A. McCarthy for pointing out the following:

Theorem 2. If $T^{n}=S, n$ a positive integer, $S$ an invertible spectral operator on a Banach space $X$ then $T$ is a spectral operator.

Proof. Again the proof is a slight modification of previous methods. Let $S=A+N$, where $A$ is a scalar operator and $N$ a quasinilpotent operator which commutes with $A$. Then $\left[T A^{-1 / n}\right]^{n}=S \cdot A^{-1}$ $=(A+N) A^{-1}=I+N A^{-1}$. Now since $N A^{-1}$ is quasi-nilpotent, $\sigma\left(I+N A^{-1}\right)=1$ so $\sigma\left(T A^{-1 / n}\right)$ can consist of at most the $n$th roots of unity. Now one can show by the argument of Lemma 1 that $T A^{-1 / n}$ $=\sum_{i=1}^{n} w_{i} E_{i}+Q$ where $Q$ is a quasi-nilpotent operator which commutes with all the idempotents $E_{i}, i=1, \cdots, n$. Thus $T=A^{1 / n} \sum_{i=1}^{n} w_{i} E_{i}+A^{1 / n} Q$, where the first term on the right side is a scalar operator and the second is a quasi-nilpotent operator which commutes with it.

CoRollaRy 2. If $T^{n}=S, n$ a positive integer, $S$ a spectral operator on a Banach space $x$ where zero is an isolated point of the spectrum of $S$, then $T$ is a spectral operator.

The proof requires only a slight modification of the proof of Theorem 2.

Example. We now exhibit an example to show that Theorems 1 and 2 need not be true for operators which have zero as a limit point of the spectrum. Let $H=L_{2}[0,1] \oplus L_{2}[0,1]$ with the usual Hilbert space norm. For $\left[f_{1}, f_{2}\right] \in H$ define $T\left[f_{1}(s), f_{2}(s)\right]$ $=\left[s f_{1}(s)+f_{2}(s),-s f_{2}(s)\right]$. Then elementary but tedious calculations with two $X$ two matrices show that $T$ is neither a scalar nor a spectral operator. However $T^{2}\left[f_{1}(s), f_{2}(s)\right]=\left[s^{2} f_{1}(s), s^{2} f_{2}(s)\right]$ so $T^{2}$ is clearly a normal, thus a scalar operator.

\section{BibLIOGRAPHY}

1. N. Dunford, Spectral operators, Pacific J. Math. 4 (1954), 321-354.

2. S. Foguel, Sums and products of commuting spectral operators, Ark. Mat. 8 (1957), 449-461.

3. E. Hille and R. Phillips, Functional analysis and semi-groups, Amer. Math. Soc. Colloq. Publ. Vol. 31, Amer. Math. Soc., Providence, R. I., 1957.

YALE UNIVERSITY 